\title{
EDITORIAL
}

\section{Nitric oxide and microvascular permeability: a continuing dilemma}

\author{
P. Kubes
}

In this issue of the Journal, the study by KagEYAmA et al. [1] examines the role of endogenous nitric oxide in Evans blue dye leakage from the airway microvasculature induced by inflammatory mediators that may be relevant to asthma (substance $\mathrm{P}$, leukotriene $\mathrm{D}_{4}\left(\mathrm{LTD}_{4}\right)$ and histamine). The authors demonstrate that endogenous nitric oxide (NO) plays an important role in the increased leakage of Evans blue dye following substance $\mathrm{P}$ or $\mathrm{LTD}_{4}$ administration, but not after administration of histamine. Furthermore, the authors demonstrate that the substance $\mathrm{P}$ and $\mathrm{LTD}_{4}$-induced rise in Evans blue dye leakage is increased via endogenous NO in the trachea and main bronchi, but not in the intrapulmonary airways, suggesting differential regulation of transvascular protein flux in anatomically different parts of the lung airway microvasculature. The authors also demonstrate that the substance P-induced Evans blue dye leakage was inhibitable by a soluble guanylate cyclase inhibitor (LY83583). Interestingly, this analogue primarily inhibited the protein leakage in intrapulmonary airways, with lesser effects in the main bronchi and essentially no effects in the trachea. Clearly, the pattern for the NO inhibition and cyclic guanosine monophosphate (cGMP) inhibition were different.

The technique used in this study, protein leakage out of the microcirculation, is a commonly used index of vascular permeability. However, one could argue that an increase in this parameter may not necessarily be related to microvascular permeability per se. A decrease in microvascular hydrostatic pressure as a result of potent arteriolar vasocontriction could account for a decrease in protein leakage out of the microcirculation. Another mechanism by which vascular protein leakage could be reduced is via a decrease in surface area. A derecruitment of capillaries and post-capillary venules would decrease the surface area for protein and fluid filtration out of the microvasculature, thereby giving the impression that a vasoconstrictor was actually decreasing microvascular permeability. Measures of systemic blood pressure do give insight into total vascular resistance, but do not provide information regarding blood pressure or surface area alterations in a single vascular bed. Kageyama et al. [1] clearly recognize this limitation and are careful not to exclude potential pulmonary airway microvascular haemodynamic alterations as contributing to the results obtained in this study. Neverthe-

Correspondence: P. Kubes, Dept of Physiology and Biophysics, University of Calgary, Faculty of Medicine, 3330 Hospital Drive N.W., Calgary, Alberta, Canada. less, these issues always complicate the interpretation of endogenous NO as a mediator of endothelial permeability in response to various stimuli.

In 1995, I reviewed the literature regarding the role of nitric oxide and microvascular permeability alterations in an attempt to try and find a commonality among similar observations and an explanation for discrepant results from numerous laboratories [2]. I was by no means successful in resolving the controversy, although a few trends became apparent. First, tissues such as skin, cheek pouch and paw microvasculature responded to histamine, bradykinin and substance $\mathrm{P}$ with increased plasma protein leakage and this was inhibitable by NGnitro-L-arginine methyl ester (L-NAME) [3-6]. On the other hand L-NAME further exacerbated plasma protein leakage in response to platelet activating factor (PAF), endothelin-1 or serotonin in kidney, stomach and intestine as well as the paw [3,7-9]. Therefore, nitric oxide could increase plasma protein leakage in response to histamine and decrease the protein leakage in response to serotonin in the same species and same organ. The two opposing observations become extremely difficult to reconcile if one interprets these results as a direct effect of NO on endothelial permeability. However, because the authors were examining oedema as an end point, this result might be entirely attributable to differing alterations in blood flow. Simply constricting the paw would dramatically reduce transvascular fluid and protein flux and would be likely to reduce oedema formation. Therefore, it would be most interesting to know, in the study by KaGEYAMA et al. [1], what effects histamine, $\mathrm{LTD}_{4}$ and substance $\mathrm{P}$ have on blood flow through the pulmonary airway vasculature and what effect L-NAME has on blood flow in the vasculature treated with these substances.

In the study by KageYama et al. [1], the results for substance $\mathrm{P}$ in the lung are in line with observations made by others in the rat skin [6]. Substance P increases protein leakage via nitric oxide. However, the lack of effect of L-NAME with histamine in the lung is in contrast to other observations in the paw and cheek pouch $[3,4]$. This latter observation raises the possibility that each organ including the lung has its own mediator profile that releases NO to increase plasma protein leakage. The data to date in the lung, from other investigators, are not entirely in agreement with this study in terms of the role of NO. For example, FiLEP and FoldES-FiLEP [8] reported that L-NAME alone consistently increased Evans blue dye leakage in the trachea, upper and lower bronchi, but not the pulmonary parenchyma. Furthermore, 
L-NAME exacerbated Evans blue dye leakage in response to endothelin-1 in the pulmonary parenchyma, upper and lower bronchi, but not the trachea [7]. On the other hand, Evans blue dye leakage was enhanced by L-NAME in response to PAF in trachea, bronchus and pulmonary parenchyma [8] consistent with the observation by KAGEYAMA et al. [1] that different parts of the pulmonary airway vasculature may respond differently. However, the results of KageYama et al. [1] and FILEP and FOLDES-FILEP [8] are diametrically opposed for NO. Perhaps this is reflective of potential differences of LNAME on the pulmonary airway vasculature of the rat versus the guinea pig, or of PAF used by FILEP and Foldes-FILEP [8] versus substance P used by KageYAMA et al. [1]. Again, the possible effect on haemodynamic parameters needs to be considered when interpreting these data.

ERJEFALT and co-workers [10] circumvented many of the aforementioned haemodynamic problems by injecting $5 \mathrm{~nm}$ gold particles into guinea pigs. These particles were unable to pass paracellulary out of the vasculature and into the interstitium under normal conditions, but could do so if the endothelium retracted. Topical application of L-NAME to the tracheal mucosa greatly increased exudation of these particles into the extravascular space surrounding subepithelial venules. ${ }^{125} \mathrm{I}$-albumin also increased in the interstitial space. This work suggests that inhibition of NO synthase did invoke an increase in microvascular permeability in the airway vasculature. Interestingly, in this study total tissue content of ${ }^{125} \mathrm{I}-$ albumin did not increase after topical L-NAME administration despite the profound increase in microvascular permeability. The authors postulated that L-NAME causes such a profound vasocontriction that the intravascular pool of ${ }^{125} \mathrm{I}$-albumin was reduced as much as or more than the extravascular tissue pool of accumulating ${ }^{125} \mathrm{I}$-albumin. However, it is unlikely that the reduction in Evans blue dye leakage with L-NAME reported by KaGEYAMA et al. [1] could be explained by a reduced intravascular pool of Evans blue dye per se; if indeed there was potent vasocontriction then reduced hydrostatic pressure or alterations in surface area could contribute to the observations reported.

In summary, the study by KAGEYAMA et al. [1] demonstrates that the same mediator, in this case nitric oxide, can have very different effects in different parts of the airway microvasculature and, as evidenced by the LY83583 data, nitric oxide may also work by either a cyclic guanosine monophosphate dependent (main bronchi) or independent event (trachea). A further series of studies examining blood flow through individual microvascular beds would permit greater interpretation of data that are clearly relevant to the asthma literature.

\section{References}

1. Kageyama N, Miura M, Ichinose M, et al. Role of endogenous nitric oxide in airway microvascular leakage induced by inflammatory mediators. Eur Respir $J$ 1996; 9: 13-19.

2. Kubes P. Nitric oxide affects microvascular permeability in the intact and inflamed vasculature. Microcirc 1995; 2: 235-244.

3. Oyanagui Y, Sato S. Histamine paw edema of mice was increased and became $\mathrm{H}_{2}$-antagonist sensitive by coinjection of nitric oxide forming agents, but serotonin paw edema was decreased. Life Sciences 1993; 52: 159_ 164.

4. Mayhan WG. Nitric oxide accounts for histamine-induced increases in macromolecular extravasation. Am J Physiol 1994; 266: H2369-H2373.

5. Paul W, Douglas GJ, Lawrence L, et al. Cutaneous permeability responses to bradykinin and histamine in the guinea-pig: possible differences in their mechanism of action. Br J Pharmacol 1994; 111: 159-164.

6. Hughes SR, Williams TJ, Brain SD. Evidence that endogenous nitric oxide modulates oedema formation induced by substance P. Eur J Pharmacol 1990; 191: 481-484.

7. Filep JG, Foldes-Filep E, Rousseau A, Sirois P, Fournier A. Vascular responses to endothelin-1 following inhibition of nitric oxide synthesis in the conscious rat. $\mathrm{Br}$ J Pharmacol 1993; 110: 1213-1221.

8. Filep JG, Foldes-Filep E. Modulation by nitric oxide of platelet-activating factor-induced albumin extravasation in the conscious rat. Br J Pharmacol 1993; 110: 13471352.

9. Kubes P, Reinhardt PH, Payne D, Woodman RC. Excess nitric oxide does not cause cellular, vascular or mucosal dysfunction in the cat small intestine. Am J Physiol 1995; 269: G34-G41.

10. Erjefalt JS, Erjefalt I, Sundler F, Persson CGA. Mucosal nitric oxide may tonically suppress airways plasma exudation. Am J Respir Crit Care Med 1994; 150: 227-232. 